Document downloaded from:

http://hdl.handle.net/10251/145999

This paper must be cited as:

Villegas-Villalobos, S.; Diaz, L.; Vilariño, G.; Vallés Lluch, A.; Gómez-Tejedor, J.; Valero, M. (14-0). Effect of an organotin catalyst on the physicochemical properties and biocompatibility of castor oil-based polyurethane/cellulose composites. Journal of Materials Research. 33(17):2598-2611. https://doi.org/10.1557/jmr.2018.286

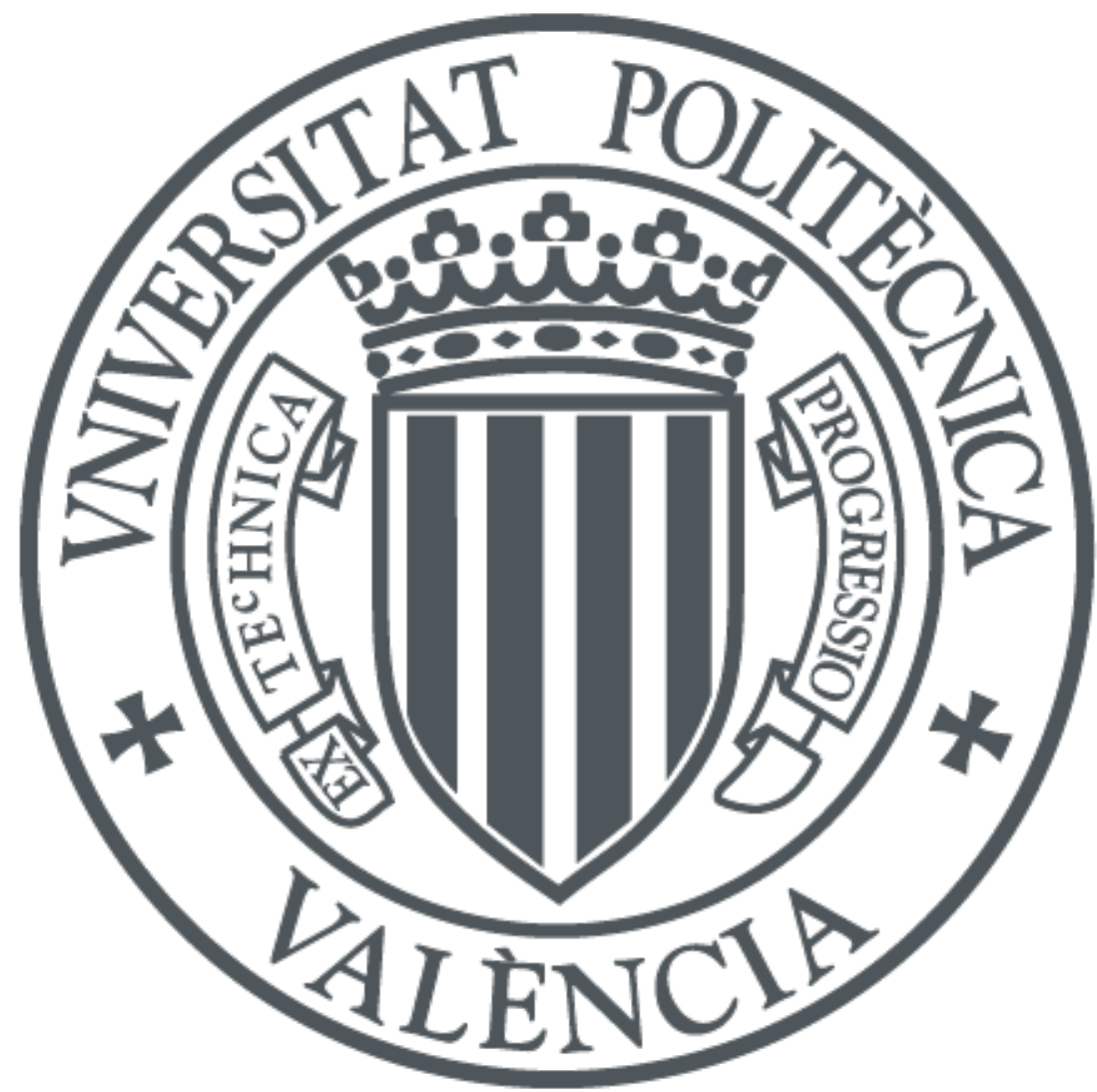

The final publication is available at

https://doi.org/10.1557/jmr.2018.286

Copyright Cambridge University Press (Materials Research Society)

Additional Information 


\title{
Effect of an organotin catalyst on the physicochemical properties and biocompatibility of castor oil-based polyurethane/cellulose composites
}

Journal of Materials Research, 2018, 33 (17): 2598-2611. DOI: 10.1557/jmr.2018.286

Santiago Villegas-Villalobos

Master in Process Design and Management, Research Group on Energy, Materials and Environment, Faculty of engineering, Universidad de La Sabana, Chía 140013, Colombia.

Luis E. Díaz

Bioprospecting Research Group, Universidad de La Sabana, Chía 140013, Colombia.

Guillermo Vilariño-Feltrer

Centre for Biomaterials and Tissue Engineering, Universitat Politècnica de València, Valencia 46022, Spain.

Ana Vallés-Lluch and José A. Gómez-Tejedor

Centre for Biomaterials and Tissue Engineering, Universitat Politècnica de València, Valencia 46022, Spain.

Biomedical Research Networking Centre in Bioengineering, Biomaterials and Nanomedicine (CIBER-BBN), Valencia 46022, Spain.

Manuel F. Valero

Research Group on Energy, Materials and Environment, Faculty of Engineering, Universidad de La Sabana, Chía 140013, Colombia.

\begin{abstract}
Polyurethane/cellulose composites were synthesized from castor-oil-derived polyols and isophorone diisocyanate using dibutyltin dilaurate (DBTDL) as catalyst. Materials were obtained by adding $2 \%$ cellulose in the form of either microcrystals $(20 \mu \mathrm{m})$ or nanocrystals obtained by acid hydrolysis. The aim was to assess the effects of filler particle size and the use of a catalyst on the physicochemical properties and biological response of these composites. The addition of the catalyst was found to be essential to prevent filler aggregations and to enhance the tensile strength and elongation at break. The cellulose particle size influenced the composite properties, as its
\end{abstract}


Journal of Materials Research, 2018, 33 (17): 2598-2611. DOI: 10.1557/imr.2018.286

nanocrystals heighten hydrogen bond interactions between the filler surface and polyurethane domains, improving resistance to hydrolytic degradation. All hybrids retained cell viability, and the addition of DBTDL did not impaired their biocompatibility. The samples were prone to calcification, which suggests that they could find application in the development of bioactive materials.

Key Words: polymer, composite, organometallic-catalyst.

\section{INTRODUCTION}

Various types of diseases and injuries can lead to tissue damage, loss of organ function, fractures and disfigurations. Treatment for these types of problems demands biomaterials that promote recovery of proper function in various regions of the body. Use of polyurethanes as biomaterials has increased due to their excellent mechanical properties and biocompatibility. ${ }^{1}$ For this reason, multiple biomedical devices are designed starting from polyurethanes of various origins according to the requirements of each application. ${ }^{2}$ Nevertheless, biomaterials that combine the properties of polyurethanes with new features deserve more attention, as these materials could further enable treatment of different physical disorders. ${ }^{3}$

In recent years, the incorporation of fillers into polymeric matrices has been explored, and the results show improvements in the properties of the materials and even yielding materials with completely new properties. ${ }^{4}$ In particular, the use of cellulose as reinforcing material in polymer matrices is an emerging field of study. For instance, adding cellulose nanofibers extracted from pineapple leaves to a polyurethane matrix made of di-p-phenyl-diisocyanate, polycaprolactone diol $\left(M_{n}=530\right)$ and 1,4-butanediol improved significantly the properties of the material. The composite showed an increase in Young's modulus $\left(E^{\prime}\right)$ and tensile strength $(\sigma)$ of $2600 \%$ and $300 \%$, respectively, after the addition of $5 \%$ nanofibers. ${ }^{5}$ The material withstood 12 years of cyclic use in accelerated fatigue tests and in vivo implantation in rats. Besides, this material is suitable for use in cardiac valves and vascular implants because it maintains good hemodynamic properties.

Indeed, there are several characteristics that make cellulose an interesting reinforcing material, such as its high modulus of elasticity, low density $\left(\approx 1.6 \mathrm{~g} / \mathrm{cm}^{3}\right)$, low cost and the opportunity to incorporate a renewable carbon source, in particular, in the production of polyurethanes. ${ }^{6}$ Introducing vegetable oils in the synthesis of polyurethanes is another approach to producing environmentally friendly materials, as polyols derived from vegetable oils can replace those from fossil sources. ${ }^{7}$ Among the many vegetable oils that can be used to synthesize polyurethanes, castor 
Journal of Materials Research, 2018, 33 (17): 2598-2611. DOI: 10.1557/imr.2018.286

oil is the most attractive, as it is the only natural oil polyol produced directly by nature. ${ }^{8}$ Approximately $90 \%$ of castor oil fatty acids is ricinoleic acid, which has a hydroxyl functionality at the carbon-12 position; ${ }^{9}$ therefore, no chemical transformation is necessary for its incorporation in polyurethane production.

Some authors have researched the use of cellulose in the form of either micro- or nanocrystals as reinforcing material in castor oil-based polyurethane matrices using different types of diisocyanates. In sum, these studies have found that cellulose affects the thermal and mechanical properties by increasing thermal stability and $E^{\prime}$, while diminishing elongation at break $(\varepsilon \%)$. However, a significant difference in these studies regards the tensile strength of the materials: while the composites synthesized with microcrystalline cellulose showed a decrease in tensile strength, ${ }^{10}$ those that integrated nanocrystals obtained by acid hydrolysis showed an increase. ${ }^{11-14}$ Regarding the composition of the materials, most of them presented optimum behavior with cellulose loads of approximately 1-2 wt.\%, whereas higher amounts of filler lead to aggregation. Only in one of the aforementioned studies a catalyst was used in the synthesis: dibutyltin dilaurate (DBTDL), which provided the greatest improvement in the mechanical properties with an increase in $\sigma$ of $125 \%$ and a small decrease in $\varepsilon \%$ of $2.9 \%{ }^{13}$

Of the various catalysts used in polyurethane synthesis, DBTDL the most efficient, along with iron acetylacetonate. ${ }^{15}$ Nevertheless, most of the commonly used catalysts in the synthesis of urethanes are known to be toxic, which is the case for DBTDL; ${ }^{16}$ hence, it may negatively affect the biocompatibility of polyurethanes. Some authors have though proposed different purification methods to overcome this inconvenience. ${ }^{17-19}$

Besides, harmful particles leaching from the matrix and possible enzymatic degradation products may also diminish the biocompatibility of the materials during use in biomedical field. ${ }^{20}$ However, none of the aforementioned studies evaluating the use of cellulose in castor oil-based polyurethanes report any cytotoxicity testing. As stated, the cited works show that cellulose is a promising reinforcing material for obtaining stable and biocompatible materials, which could be of tremendous value to the biomedical industry. Nevertheless, they focus on improvements in the properties of the composites and overlook the evaluation of their biological responses. The purpose of this study is thus to assess the role of filler particle size and the use of an organotin catalyst on the physicochemical and biological properties of materials synthesized from cellulose crystals, castor oil (from Ricinus communis) and isophorone diisocyanate. The requirement of a rinsing 
Journal of Materials Research, 2018, 33 (17): 2598-2611. DOI: 10.1557/imr.2018.286

protocol after synthesis to eliminate eventual unreacted raw materials and catalyst traces and subsequently diminish the risk of releasing harmful particles is also ascertained.

\section{MATERIALS AND METHODS}

\section{A. Cellulose nanocrystals isolation}

Cellulose nanocrystals (CNC) were obtained by acid hydrolysis of microcrystalline cellulose (MCC; Sigma-Aldrich). MCC with an average size of $20 \mu \mathrm{m}$ (FIG. 1 (a)) was mixed with deionized water and sulfuric acid $\left(\mathrm{H}_{2} \mathrm{SO}_{4}\right.$; Scharlau), achieving a final acid concentration of $64 \% \mathrm{v} / \mathrm{v}$ and maintaining a ratio of cellulose to acid medium of 1:10 w/v. Following the methodology proposed in, ${ }^{21}$ the reaction was performed at $44^{\circ} \mathrm{C}$ for $2 \mathrm{~h}$, after which distilled water was added to quadruple the volume. The suspension was then rinsed with deionized water by means of repeated centrifuge cycles at 5000 $\mathrm{rpm}$ for $4 \mathrm{~min}$. As the supernatant became turbid, the washing process was stopped, the supernatant discarded, and the precipitate neutralized by adding $0.2 \mathrm{M}$ sodium hydroxide $(\mathrm{NaOH}$; Scharlau). The nanocrystals in the aqueous phase were washed with acetone for three days following the protocol reported in ${ }^{22}$ with slight modifications, i.e., the solvent was changed twice per day by adding a volume of acetone equal to the initial volume of the aqueous dispersion of the nanocrystals, and the organic phase was stirred regularly to facilitate the solvent change. CNCacetone gels with nanocrystal concentrations between $11-15 \%$ were obtained.

\section{B. Synthesis of polyurethane/cellulose composites}

Castor oil (CO; Ciacomeq S.A.S.) with a hydroxyl number of $160 \mathrm{mg} \mathrm{KOH} / \mathrm{g}$ and isophorone diisocyanate (IPDI; Alfa Aesar) were used as-purchased. Samples of neat castor-oil based polyurethane (COPU) and with 2 wt.\% MCC and CNC (COPU/MCC and COPU/CNC) were synthesized. Another set of samples was synthesized using DBTDL (Alfa Aesar) as catalyst to accelerate curing times (COPU/Cat., COPU/MCC/Cat. and COPU/CNC/Cat.). Composites were synthesized via in situ polymerization. For the $\mathrm{CNC}$ composite sample synthesis, $\mathrm{CO}$ was mixed with $\mathrm{CNC}$-acetone gels. To avoid agglomeration, the mixture was blended in a VCX-750 ultrasonic processor (Sonics) at $80 \%$ amplitude for $6 \mathrm{~min}$. Acetone was removed by evaporation under reduced pressure, and the mixture was sonicated for additional $4 \mathrm{~min}$ and then heated to $60{ }^{\circ} \mathrm{C}$. When the set temperature was reached, IPDI was added; the mixture was stirred for additional $5 \mathrm{~min}$ and vacuumed to remove bubbles. The amount of IPDI added was calculated so as to maintain a $[\mathrm{NCO}] /[\mathrm{OH}]$ ratio of 1 , including only the hydroxyl groups present in CO. For the materials synthesized without acetone gel, 
Journal of Materials Research, 2018, 33 (17): 2598-2611. DOI: 10.1557/imr.2018.286

the reduced pressure evaporation process was omitted, and sonication was applied for 10 min before adding IPDI to the CO/MCC mixture. For the samples synthesized with catalyst, DBTDL (68 $\mu L$ ) was added and stirred for 2 min after bubbles were removed. Finally, the samples were poured into steel molds and cured at $105^{\circ} \mathrm{C}$ for $12 \mathrm{~h}$.

\section{Rinsing protocol}

Three films of each composition, weighing between 250 and $300 \mathrm{mg}$, were rinsed with tetrahydrofuran (THF; Scharlau) for 1 day. The solvent was progressively changed to ethanol (Scharlau) in a four-steps process. Samples were then rinsed in pure ethanol for 12 and $24 \mathrm{~h}$ after the first solvent change. Following the fourth day, ethanol was changed to deionized water for an additional day. Samples were next dried under vacuum at $50{ }^{\circ} \mathrm{C}$ until constant weight. A Mettler AX 205 balance (Mettler-Toledo Inc.) with a sensitivity of $0.01 \mathrm{mg}$ was used to weigh samples before and after rinses to measure any mass loss. Three parallel samples for each material type were used.

\section{Field-emission scanning electron microscopy (FE-SEM)}

Particle size determination for the cellulose crystals was performed on an AURIGA Compact fieldemission SEM (Zeiss) operating at an accelerating voltage of $2 \mathrm{kV}$ and a working distance of $4 \mathrm{~mm}$. Water suspensions of cellulose were dried on glass sample holders and coated with platinum before observation. Analysis of the micrographs was performed using the ImageJ software. Images of freeze-fractured polyurethane samples were also taken.

\section{E. Fourier transform infrared spectroscopy}

Fourier transform infrared (FTIR) spectra of the samples were collected using a Bruker PLATINUM ATR infrared spectrometer to reveal differences in the chemical interactions of hard segments (HS) and soft segments (SS) in the neat polyurethane and its composites. The spectra were measured from 400 to $4000 \mathrm{~cm}^{-1}$ at $4 \mathrm{~cm}^{-1}$ resolution.

\section{F. Density tests}

The aforementioned balance, equipped with a Mettler ME 33360 density accessory kit, was used to determine the density through Archimedes' principle by immersion in n-octane (reagent grade 98\%; Aldrich; $\left.\rho_{\mathrm{n} \text {-octano }}=0.703 \mathrm{~g} \cdot \mathrm{mL}^{-1}\right)$. Dry samples were weighed in air $\left(m_{\text {in air }}\right)$ and immersed in $\mathrm{n}$-octane ( $\left.m_{\text {in n-octane }}\right)$ at room temperature. The density, $\rho$, was calculated as the ratio of $m_{\text {in air }}$ to the volume of $\mathrm{n}$-octane displaced $\left(V_{\text {displaced }}\right)$ : 
Journal of Materials Research, 2018, 33 (17): 2598-2611. DOI: 10.1557/imr.2018.286

$\rho=\frac{m_{\text {in air }}}{V_{\text {displaced }}}=\frac{m_{\text {in air }}}{\left(m_{\text {in air }}-m_{\text {in } n-\text { octane }}\right) / \rho_{\text {n-octane }}}$

Each sample was weighed three times. Potential swelling of the samples due to immersion in $n-$ octane was analyzed before the measurements (data not shown) and considered negligible.

\section{G. Swelling in water}

Swelling in water was determined by weighing pieces with dimensions of $5 \mathrm{~mm} \times 5 \mathrm{~mm} \times 3 \mathrm{~mm}$ (length, width, thickness) of the samples immersed in distilled water at $37^{\circ} \mathrm{C}$. Measurements were done in triplicate at 24,48 and $144 \mathrm{~h}$. The percentages of swelling (swelling water $_{\text {) }}$ of each sample was calculated as the ratio between the water absorbed by the sample $\left(m_{\text {water }}\right)$ and the initial mass of the dry sample:

swelling water $=\frac{m_{\text {water }}}{m_{\text {dry sample }}}=\frac{\left(m_{\text {wet sample }}-m_{\text {dry sample }}\right)}{m_{\text {dry sample }}} \times 100$

where $m_{\text {dry sample }}$ and $m_{\text {wet sample }}$ are the weights of the sample before and after immersion in water, respectively.

\section{H. Wettability tests}

The water contact angles (WCAs) were measured by optical contact angle measurements and drop shape analyses using an OCA20 device by Data Physics. Four $5 \mu \mathrm{L}$ drops were placed on the surfaces of dry samples in sessile drop mode. The WCA reported for each sample is the average of the measurements from the right and left sides for each drop.

\section{Thermogravimetric analysis (TGA)}

The thermal stability of the samples was assessed in a Mettler Toledo TGA/DSC 2 device. Approximately $7 \mathrm{mg}$ of sample were heated from 30 to $600{ }^{\circ} \mathrm{C}$ at a heating rate of $10^{\circ} \mathrm{C} / \mathrm{min}$ under nitrogen atmosphere.

\section{J. Differential scanning calorimetry (DSC)}

DSC scans were taken using a PerkinElmer DSC 8000 to correlate the compositions and thermal properties of the samples. Specimens of approximately $5 \mathrm{mg}$ of each sample were cooled to $-80{ }^{\circ} \mathrm{C}$, stabilized for $1 \mathrm{~min}$ and next heated to $200^{\circ} \mathrm{C}$ at $20^{\circ} \mathrm{C} / \mathrm{min}$ in a sealed aluminum pan under nitrogen atmosphere. 
Journal of Materials Research, 2018, 33 (17): 2598-2611. DOI: 10.1557/imr.2018.286

\section{K. Mechanical tests}

The elastic moduli, E', and deformation percentage, $\varepsilon \%$, were evaluated using an EZ-LX (Shimadzu) universal testing machine with a load cell of $5 \mathrm{kN}$ and a frame displacement speed of $10 \mathrm{~mm} / \mathrm{min}$. Four specimens of each sample with dimensions of $40 \mathrm{~mm} \times 6 \mathrm{~mm} \times 3 \mathrm{~mm}$ (length, width, thickness) were used for these tests.

\section{Hydrolytic and enzymatic degradation assays}

Hydrolytic and enzymatic degradation assays were performed in parallel to investigate the stability of the samples in aqueous media. Specimens between 150-200 mg with dimensions of $7 \mathrm{~mm} \times 7$ $\mathrm{mm} \times 3 \mathrm{~mm}$ (length, width, thickness) were used. Each one was immersed in $1.5 \mathrm{~mL}$ of medium. Hydrolytic degradation was performed in phosphate buffered saline (PBS) for 3 months at $37^{\circ} \mathrm{C}$.

Enzymatic degradation was induced with esterase from porcine liver (Sigma-Aldrich) in phosphate buffered saline (PBS) at $37^{\circ} \mathrm{C}$ for 21 days. Samples were immersed in $1.5 \mathrm{~mL}$ of enzyme solution (10 units $/ \mathrm{mL}$ ) and removed for analysis every seven days. To preserve enzymatic activity, the degradation medium was changed every $48 \mathrm{~h}$. Four replicates were used for each data point. Poly $(\varepsilon-$ caprolactone) ( $\mathrm{PCL}$ ) was used as a reference material for the enzymatic degradation assays and samples immersed in PBS with no enzyme served as negative controls.

\section{In vitrocalcification assay}

Golomb and Wagner solution was used to simulate in vitro calcification conditions. The solution consisted of $3.87 \mathrm{mmol}$ of calcium chloride $\left(\mathrm{CaCl}_{2}\right), 2.32 \mathrm{mmol}$ of dipotassium phosphate $\left(\mathrm{K}_{2} \mathrm{PHO}_{4}\right)$ and 0.05 mol of Tris buffer in 1 liter of water. Samples were in contact with the calcification medium for 4 weeks, with weekly solution changes as described in. ${ }^{23}$ The presence of calcium deposits on the sample surfaces was assessed using the aforementioned FE-SEM. Energy dispersive X-ray spectroscopy (EDS) was conducted to determine the relative amounts of calcium and phosphorus on the samples, which were previously coated with carbon for these analyses. Titanium was used as optimization standard.

\section{N. Cell viability assay}

In vitro cell viability was evaluated using L929 fibroblasts (mouse C3H/An connective tissue; SigmaAldrich) in direct contact with the samples. Cubic samples of the materials were exposed to UV radiation for $20 \mathrm{~min}$ on each side to minimize the bacterial load of the material. Specimens with dimensions of $7 \mathrm{~mm} \times 7 \mathrm{~mm} \times 3 \mathrm{~mm}$ (length, width, thickness) were used. Cells were pre-cultured 
Journal of Materials Research, 2018, 33 (17): 2598-2611. DOI: 10.1557/imr.2018.286

for $24 \mathrm{~h}$ at $37{ }^{\circ} \mathrm{C}$ and $5 \% \mathrm{CO}_{2}$ in a 96-well plate (10,000 cells per well) with Dulbecco's Modified Eagle's Medium (DMEM, Fisher) supplemented with 10\% fetal bovine serum (FBS; Innoprot) and 1\% penicillin-streptomycin (P/S; Innoprot). Subsequently, the specimens were introduced and left in contact with the cells for $24 \mathrm{~h}$ under the same pre-culture conditions.

For cell viability quantification, the medium was removed and $100 \mu \mathrm{L}$ of 3-(4,5-dimethylthiazol-2yl)-2,5-diphenyltetrazolium bromide (MTT; 10\% in DMEM without phenol red) were added to each well. After $2 \mathrm{~h}$ of incubation, the supernatant was discarded, and isopropanol was added to break cell membranes and allow the formazan salts to solubilize for $10 \mathrm{~min}$. Optical density (OD) measurements were performed with a PerkinEImer VICTOR ${ }^{3}$ plate reader at a wavelength of 570 $\mathrm{nm}$. All tests were performed in quadruplicate, and wells cultured without samples and with latex served as positive and negative controls, respectively. The percentage of cell proliferation was calculated using the following equation:

Cell viability $=\frac{O D_{\text {Sample }}-O D_{\text {Blank }}}{O D_{\text {Positive control }}-O D_{\text {Blank }}} \times 100 \%$

\section{O. Statistical analysis}

The results were analyzed using analysis of variance (ANOVA), and the criterion for significant differences was set to be $\alpha<0.05$. The least significant difference (LSD) post hoc test (confidence degree of 0.95 ) was selected for the multiple mean comparison. For the enzymatic degradation assay, comparisons of the means were performed using the Student's t-test. Data were processed with the STATGRAPHICS ${ }^{\circledR}$ Centurion XVII professional package (Statgraphics Technologies, Inc.). Experiments were conducted using at least three independent replicates. The results are reported as mean \pm standard deviation (SD).

\section{RESULTS AND DISCUSSION}

\section{A. Composite morphologies and physicochemical structures}

The SEM images of purchased MCC and CNC obtained by acid hydrolysis are shown in FIG. 1 (a) and FIG. 1 (b), respectively. CNC particles were highly aggregated and had a needle-like morphology with an average length of $294 \pm 72 \mathrm{~nm}$ a diameter of $29.3 \pm 2.8 \mathrm{~nm}$ and an aspect ratio (L/D) of 10. A high aspect ratio reduces the critical volume fraction that is necessary to form a percolated network of reinforcing material, which enhances mechanical strength and modulus. ${ }^{24}$ FIG. 1 (c)-FIG. 1 (e) show SEM images of the surfaces of COPU/Cat. and composite specimens that were freeze-fractured 
under liquid nitrogen. The surface of the neat polyurethane was homogeneous, indicating a proper interpenetration of the phases. ${ }^{25}$ In the MCC composites, occasional particles of cellulose trapped in the polymer matrix were observed, whereas the remainder of the surface appeared to be as homogeneous as neat polyurethane. Conversely, the CNC composite surface looked deflected and uneven, which could indicate a different energy dissipating mechanism within the matrix and the CNC. ${ }^{14}$ The roughness appeared to be uniform on the surface of the material, indicating a regular distribution of the reinforcement material within the polyurethane matrix. Materials synthesized without catalyst showed analogous behavior.

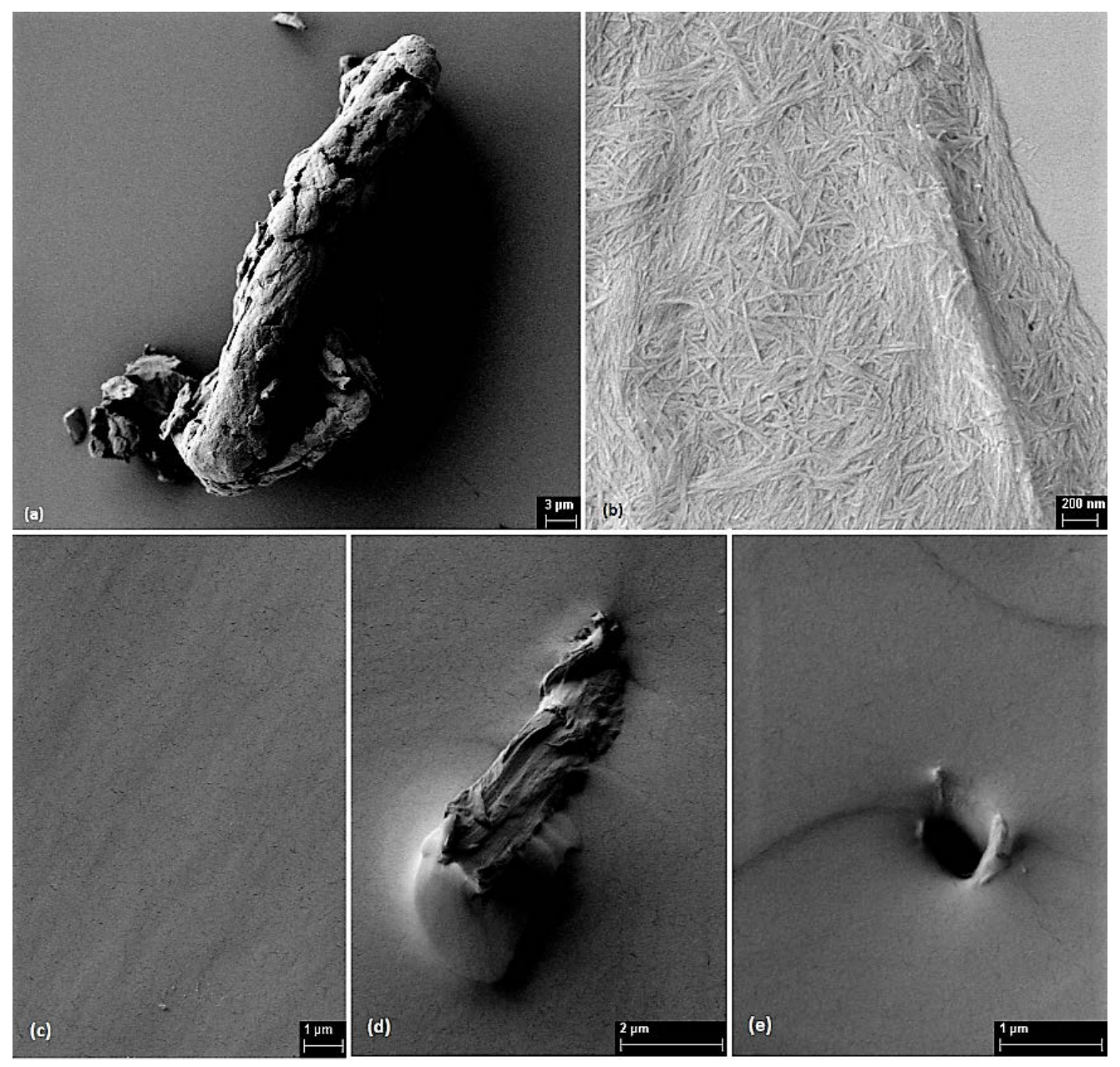

FIG. 1. SEM images of (a) microcrystalline cellulose- $1.2 \mathrm{KX}$, (b) cellulose nanocrystals- $20.0 \mathrm{KX}$, and freeze-fracture surfaces under liquid nitrogen of (c) COPU/Cat.- $4.5 \mathrm{KX}$, (d) COPU/MCC/Cat.- $6.0 \mathrm{~K}$ $\mathrm{X}$ and (e) COPU/CNC/Cat.- $12.0 \mathrm{KX}$. 
Journal of Materials Research, 2018, 33 (17): 2598-2611. DOI: 10.1557/imr.2018.286

The IR spectra for neat polyurethanes synthesized with and without catalyst are shown in FIG. 2 (a). A signature peak can be observed at $1730 \mathrm{~cm}^{-1}$ and a broad band at $3330-3340 \mathrm{~cm}^{-1}$ from the $\mathrm{C}=0$ and $\mathrm{NH}$ stretching vibrations respectively, of urethane. ${ }^{13}$ Additionally, the absence of the characteristic isocyanate peak at $2200 \mathrm{~cm}^{-1}$ indicates that IPDI was completely consumed in the catalyzed and non-catalyzed polymerization reactions. Peaks between $2800-3000 \mathrm{~cm}^{-1}$ correspond to $\mathrm{CH}_{2}$ and $\mathrm{CH}_{3}$ symmetric and asymmetric stretching, which are characteristic of bio-based polyurethanes ${ }^{26}$, while the peak at $1230 \mathrm{~cm}^{-1}$ corresponded to $\mathrm{CH}_{2}$ of aliphatic chains in polyurethane..$^{25}$ 
Journal of Materials Research, 2018, 33 (17): 2598-2611. DOI: 10.1557/imr.2018.286
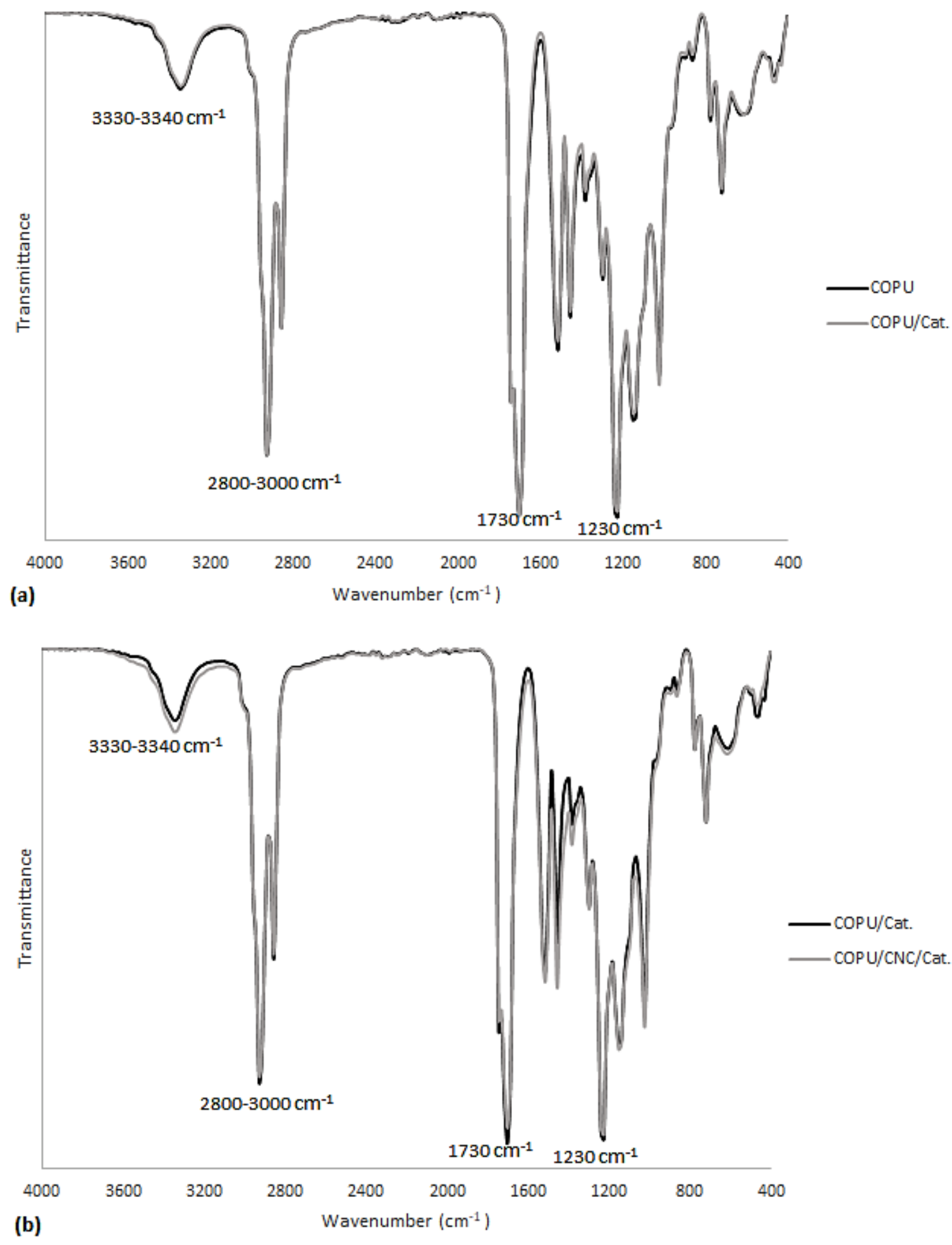

FIG. 2. IR spectra of (a) neat polyurethanes, with and without catalyst, and (b) neat polyurethane with catalyst and its CNC composite. 
Journal of Materials Research, 2018, 33 (17): 2598-2611. DOI: 10.1557/imr.2018.286

The cellulose loading used in the synthesized materials did not result in new peaks, as shown in FIG. 2 (b) where the IR spectra of neat polyurethane COPU/Cat. and the COPU/CNC/Cat. composites are be compared. Nevertheless, a difference in the magnitude of the peak at $3330-3340 \mathrm{~cm}^{-1}$ was observed. This difference could indicate a change in the polyurethane phase separation due to a hydrogen bonds interaction between polyurethane segments and cellulose in the COPU/CNC/Cat. composites. Polyurethane is known to segregate into both hard segment (HS)-rich domains that consist of urethane and are responsible for physical cross-linking, and soft segment (SS)-rich domains that consist of polyol chains (the major constituent by mass), which gives the material its elastomeric performance. ${ }^{27}$ In neat polyurethane, $\mathrm{NH}$ in the HS domains serves as proton donor, whereas $\mathrm{C}=\mathrm{O}$ from the HS and SS domains serves as proton acceptor. ${ }^{28}$ The addition of cellulose could lead to new hydrogen bond interactions between $\mathrm{OH}$ groups on the surface of cellulose and $\mathrm{C}=\mathrm{O}$ ones, with $\mathrm{C}=\mathrm{O}$ dissociating its original hydrogen bonds with $\mathrm{NH}$, which gives the soft segments more freedom to arrange into domains. ${ }^{13}$ These changes in the polyurethane phase structure and the strong interactions between cellulose and the matrix evidenced in the SEM images could affect the mechanical and thermal properties of the material, as discussed later.

Samples were rinsed to remove non-crosslinked chains, unreacted raw materials, filler particles and catalyst residues, which can affect material biocompatibility. Samples swell significantly in THF, becoming very fragile while submerged and shrinking abruptly when the solvent was changed, so the change to ethanol was done progressively. Once the samples were washed with water, they shrank to their initial shape, recovering their rubbery consistency. The mass loss after rinsing depended exclusively on the use of a catalyst, increasing to $1.51 \pm 0.23 \%$ for the non-catalyzed samples compared with $1.08 \pm 0.06 \%$ for the catalyzed samples. None of the synthesized composites, with or without catalyst, showed significantly different mass losses $(\alpha>0.05)$ compared with neat polyurethanes, suggesting that the cellulose particles (MCC and CNC) were properly integrated in the matrix and filler leaching was unlikely.

\section{B. Degree of crosslinking and hydrophilic behavior of composites}

The type of cellulose used as reinforcement material and the addition of DBTDL as catalyst had a statistically significant effect $(\alpha<0.05)$ on the density $(\rho)$ of the synthesized materials. Even though the amount of cellulose added to the matrix was very low ( $2 \mathrm{wt} . \%)$, the density increased with filler content, as shown in TABLE I, because the density of cellulose is higher than that of the PU matrix. The experimental density values for the composites were very close to the theoretical values 
Journal of Materials Research, 2018, 33 (17): 2598-2611. DOI: 10.1557/imr.2018.286

calculated using the rule of mixtures, using the experimental densities of neat polyurethanes ( $\rho_{\text {COPU }}=1.020 \mathrm{~g} \cdot \mathrm{mL}^{-1}$ and $\rho_{\text {COPU } / \mathrm{Cat} .}=1.014 \mathrm{~g} \cdot \mathrm{mL}^{-1}$ ) and taking the completely crystalline natural cellulose density as $1.5905 \pm 0.0085 \mathrm{~g} \cdot \mathrm{mL}^{-1} \cdot{ }^{29}$ As expected, the CNC-based composites had higher densities than the MCC-based ones, due to the higher crystallinity of the former. The effect of the catalyst on the density can be attributed to a less ordered polymer network in the materials where the catalyst was used, due to the accelerated polymerization reaction.

TABLE I. Experimental densities, theoretically calculated composites densities and water uptakes (WU) of neat PUs and their composites.

\begin{tabular}{cccc} 
Material & $\begin{array}{c}\text { Experimental } \\
\text { Density }\left(\mathbf{g} \cdot \mathrm{mL}^{-1}\right)\end{array}$ & $\begin{array}{c}\text { Composites Calculated } \\
\text { Density }\left(\mathbf{g} \cdot \mathrm{mL}^{-1}\right)\end{array}$ & WU (\%) \\
\hline COPU & $1.020 \pm 2 \times 10^{-4}$ & & $0.41 \pm 0.02$ \\
\cline { 3 - 3 } COPU/MCC & $1.025 \pm 3 \times 10^{-4}$ & $1.031 \pm 6 \times 10^{-3}$ & $0.74 \pm 0.04$ \\
COPU/CNC & $1.027 \pm 1 \times 10^{-4}$ & $0.76 \pm 0.02$ \\
COPU/Cat. & $1.014 \pm 15 \times 10^{-4}$ & & $0.39 \pm 0.05$ \\
COPU/MCC/Cat. & $1.024 \pm 1 \times 10^{-4}$ & $1.026 \pm 7 \times 10^{-3}$ & $0.54 \pm 0.03$ \\
COPU/CNC/Cat. & $1.026 \pm 1 \times 10^{-4}$ & & $1.09 \pm 0.05$
\end{tabular}

The hydrophilicity of neat PUs and their composites was analyzed via the water contact angles (WCA) and swelling in water. FIG. 3 (a) presents the average WCAs of the samples surfaces, which can be considered as not easily wettable, as all presented angles were greater than $90^{\circ} .{ }^{30}$ The hydrophobic behavior of the samples was reduced by the addition of cellulose and the catalyst. This behavior can be attributed to the hydrophilic character of cellulose and modifications in microphase separations and segment migrations, which are related to the surface energy. ${ }^{30}$ The filler particle size also had an effect on the surface hydrophilicity; the CNC composites had a lower hydrophobicity than the MCC composites. A smaller particle size may have increased the proportion of cellulose segments available at the surface of the material to interact with water. 


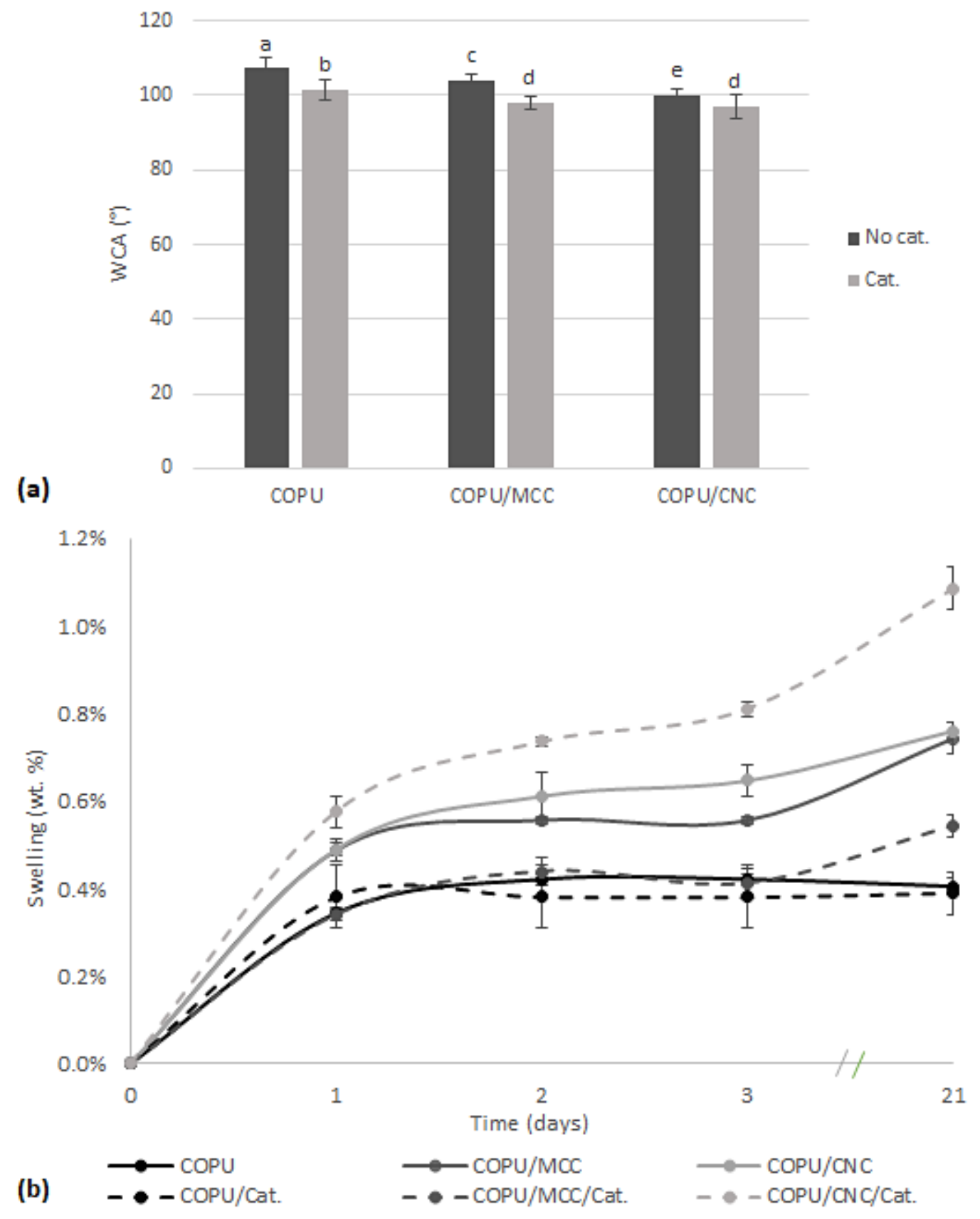

FIG. 3. (a) Water contact angles (WCAs) and (b) water sorption kinetics of neat PUs and their composites. Note: WCA data are presented as the means, and error bars represent two SD ( $n=8)$. Bars with different letters indicate statistically significant differences $(\alpha<0.05)$ according to the ANOVA and LSD post hoc test.

The water uptakes (WUs) of the samples after 21 days immersed in water are shown in TABLE I The PU neat matrix can be regarded as hydrophobic considering the amount of water absorbed (approximately $0.40 \%$ ). The evolution of sample weights during exposure to water versus time are 
Journal of Materials Research, 2018, 33 (17): 2598-2611. DOI: 10.1557/imr.2018.286

presented in FIG. 3 (b). As expected, the composites showed higher swelling due to the higher hydrophilicity of cellulose, which allowed water molecules to infiltrate the interfacial filler/matrix zone. ${ }^{31}$ The significant difference between COPU/CNC/Cat. and the other composites can be related to the formation of an interconnected filler network that promotes diffusion of water in the material, in agreement with data from literature. ${ }^{32}$ This interconnected network may not have completely formed in the other composites, either as the result of filler aggregation in the composites where the catalyst was not used or due to the low aspect ratio of the filler in the MCC composites, leading to a slower diffusion of water into the material. This absorption mechanism is clearly demonstrated in FIG. 3 (b), which shows that the neat PUs reached maximum swelling after 2 days in contact with water, while the composites kept swelling after 21 days. COPU/MCC/Cat. notably had a lower water uptake compared with the non-catalyzed composites. This lower WU was attributed to the formation of aggregates in the non-catalyzed composites, which created interstitial spaces where water accumulated.

\section{Thermal properties}

The derivative thermograms in FIG. 4 (a), calculated as the derivative of the sample weight versus the temperature measured by TGA, show three different decomposition stages for all samples. The maximum decomposition temperatures $\left(T_{d \max }\right)$ of each stage, calculated as the relative minimum of the sample weight derivative, is summarized in TABLE II, along with other thermal properties. The $1^{\text {st }}$ degradation stage, between 270 and $370{ }^{\circ} \mathrm{C}$, is known to be related to the urethane bond decomposition that occurs through three mechanisms: i) dissociation to its parent isocyanate and alcohol, ii) formation of a secondary amine and an olefin, and iii) formation of a secondary amine and carbon dioxide. ${ }^{33}$ The $2^{\text {nd }}$ stage, at approximately $380{ }^{\circ} \mathrm{C}$, corresponds to polyurethane SS pyrolytic degradation into 10-undecanoic acid and heptanal (degradation products of ricinoleic acid). ${ }^{34}$ The last stage is related to the decomposition of the polyurethane networks and other remaining structures. 

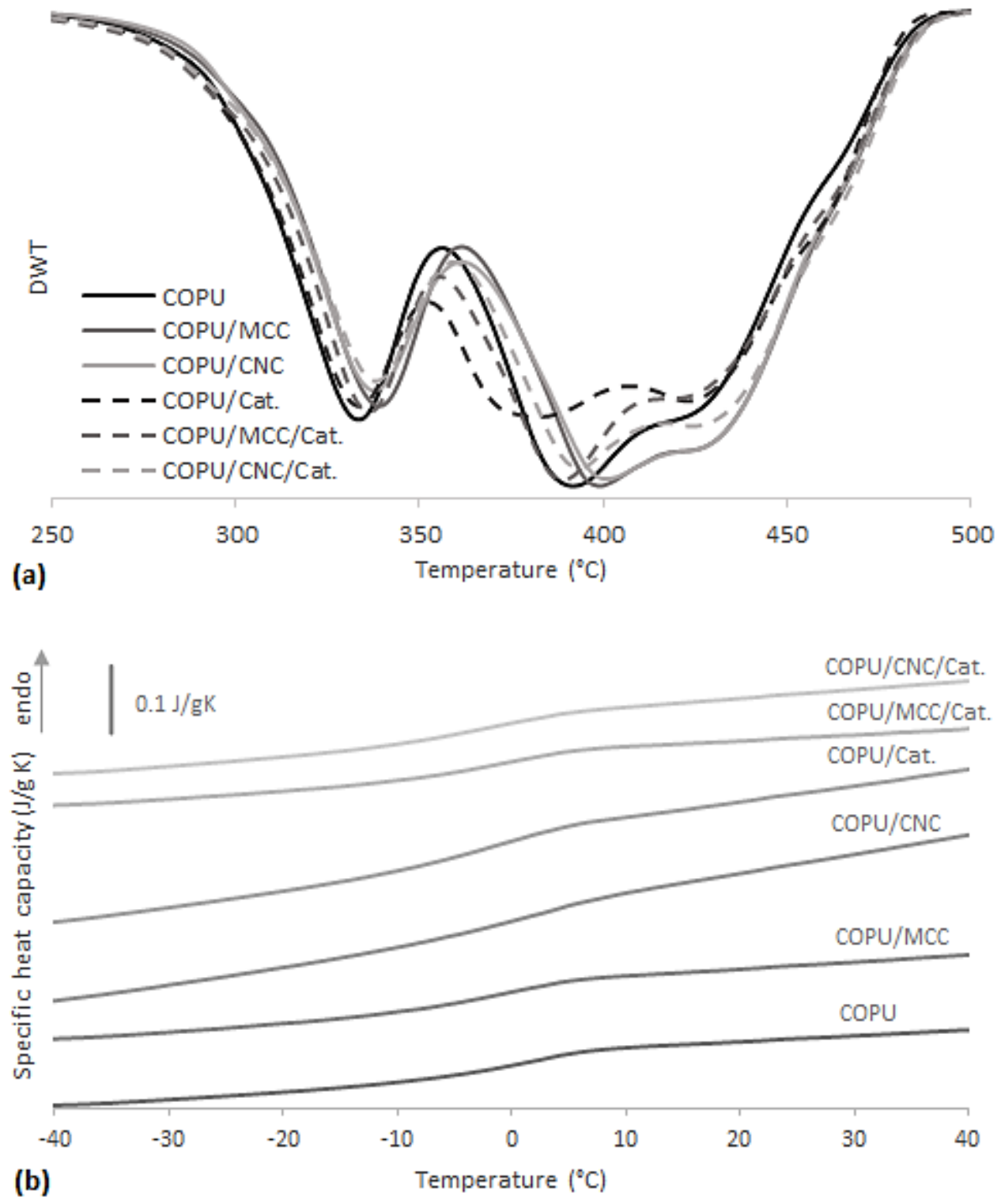

FIG. 4. (a) Weight derivatives vs. temperature obtained from TGA curves and (b) specific heat capacities of COPUs and their composites..

The $T_{d \text { max }}$ for the $1^{\text {st }}$ and $2^{\text {nd }}$ stages tends to be higher when cellulose was added. This result was attributed to the new bonds between the matrix and the hydroxyl groups on the cellulose surface, which provided higher thermal stability to the composites. ${ }^{14}$ On the other hand, the $T_{d \max }$ for the $1^{\text {st }}$ and $3^{\text {rd }}$ degradation stages were almost identical for materials with and without the catalyst. Nevertheless, in the $2^{\text {nd }}$ degradation stage, the $T_{d \max }$ seemed to be affected by the use of a catalyst, 
Journal of Materials Research, 2018, 33 (17): 2598-2611. DOI: 10.1557/imr.2018.286

being $10^{\circ} \mathrm{C}$ lower for the materials in which a catalyst was used. This result has been attributed to a catalytic effect from DBTDL on the SS-rich phase packing; a less packed domain structure leads to lower thermal stability. ${ }^{35}$ The influence of the catalyst on the thermal properties of SS-rich domains were also evident in the DSC analyses performed.

Polyurethanes with a high phase separation are known to have two glass transition temperatures $\left(\mathrm{T}_{\mathrm{g}}\right)$, one at temperatures below 0 으 $\mathrm{C}$ for the SS-rich domains and the other at temperatures above $0{ }^{\circ} \mathrm{C}$ for the HS-rich domains. ${ }^{8}$ However, the synthesized materials showed only one glass transition temperature $\left(\mathrm{T}_{\mathrm{g}}\right)$ at approximately $0^{\circ} \mathrm{C}$, as shown in the DSC normalized thermograms presented in FIG. 4 (b). The presence of only one $T_{g}$ can thus be attributed to a poor phase separation related to the curing temperature. A high curing temperature leads to HS-HS hydrogen bond disassociation, resulting in a more homogeneous structure. ${ }^{27}$ Neat castor oil polyurethanes synthesized in other studies under similar conditions, but at lower curing temperatures, show two defined $T_{g}$ at $-46.12{ }^{\circ} \mathrm{C}$ and $116.42{ }^{\circ} \mathrm{C}$ for SS-rich and HS-rich domains, respectively. ${ }^{8}$

$\mathrm{T}_{\mathrm{g}}$ values were calculated using the half $\mathrm{Cp}$ extrapolation method and are listed in TABLE II, along with the change in specific heat capacities $(\Delta C p)$. The $T_{g}$ in the DSC thermograms, assumed to be the $\mathrm{T}_{\mathrm{g}}$ for the SS-rich domains because they appear close to or below $0{ }^{\circ} \mathrm{C}$, tends to shift to lower temperatures for those materials synthesized with the catalyst compared with those without. This result can be attributed to an accelerated polymer network formation when DBTDL was used, which could cause a less organized domain structure and, as TGA analysis suggests, a less packed structure in the SS-rich domains. Subsequently, a higher $T_{g}$ was reported for COPU/CNC, suggesting that the addition of CNC resulted in higher phase separation, which combined with a slower polymerization reaction, led to higher domain separation and packing.

\section{Mechanical properties}

The neat COPUs and the composites with $2 \%$ wt. of MCC or CNC were tested under tension at room temperature (above the $T_{g}$ of materials). TABLE II shows the mechanical properties of materials, which display a typical rubbery behavior with a low tensile strength, a low Young's modulus and high elongation at break. The low strength of polyurethanes based only on $\mathrm{CO}$ as polyol result from the many dangling chains, which act as imperfections in the polymer network, and do not support the stress when loading the matrix. ${ }^{36}$ The composites without catalyst (COPU/MCC and COPU/CNC) showed little to no improvement in their mechanical properties, having the same tensile strength of neat PUs. For these materials, the lack of mechanical improvement can be related to the easier 
Journal of Materials Research, 2018, 33 (17): 2598-2611. DOI: 10.1557/imr.2018.286

aggregation of the filler. The synthesized polyurethane matrix needed long curing times, due to the low hydroxyl number of $\mathrm{CO}$; this led to the formation of agglomerates that act as defects in the composites, consequently reducing the possible reinforcing effect of the filler. Nevertheless, elongation at break was improved for COPU/CNC composite by $25 \%$, compared with neat COPU. This suggest that the CNC smaller particle size may increase the hydrogen bonds interaction between the cellulose interface and PU matrix, enhancing microphase separation.

On the other hand, the composites synthesized with the catalyst did show a statistically significant improvement $(\alpha<0.05)$ in their mechanical properties, as displayed in FIG S1. In particular, the highest tensile strength and elongation at break were found in the composite COPU/CNC/Cat., with an increase of $112 \%$ and $79 \%$, respectively, compared with neat COPU/Cat. The reinforcement effect of the cellulose fillers was attributed to the formation of a tridimensional network of cellulose particles in the PU matrix. The interaction between this network and the PU matrix probably increased the crystallinity of the hard-segments, decreasing molecular mobility and promoting rigidity, in agreement with other studies. ${ }^{37}$ Mechanical properties are therefore very sensitive to the presence of DBTDL, because it accelerates the polymerization reaction. A higher polymerization rate diminishes the time span between the mixing of cellulose during synthesis and polymer network formation, preventing agglomeration of the filler. ${ }^{38}$ The improvement in both tensile strength and elongation at break achieved in this work contrast with the results obtained in other studies that evaluated the effect of the addition of cellulose to similar PU matrices. Some authors found an increase in tensile strength and a reduction in elongation at break, ${ }^{11-14}$ while others reported a decrease in both mechanical properties ${ }^{10}$. This suggest that the proposed system allows the reinforcement of hard domains without blocking the movement of the molecular chains of the PU.

TABLE II. Thermal properties obtained from TGA ( $\left.T_{d \max }\right)$ and DSC ( $T_{g}$ and $\left.\triangle C p\right)$ analyses, and mechanical properties of PUs and their composites.

\begin{tabular}{|c|c|c|c|c|c|c|c|c|}
\hline \multirow[b]{2}{*}{ Material } & \multicolumn{3}{|c|}{$\mathrm{T}_{\mathrm{d} \max }\left({ }^{\circ} \mathrm{C}\right)$} & \multirow[b]{2}{*}{$\mathrm{T}_{\mathrm{g}}\left({ }^{\circ} \mathrm{C}\right)$} & \multirow{2}{*}{$\begin{array}{c}\Delta \mathrm{Cp} \\
(\mathrm{J} / \mathrm{g} \cdot \mathrm{K})\end{array}$} & \multirow{2}{*}{$\begin{array}{c}\text { Tensile } \\
\text { Strength (MPa) }\end{array}$} & \multirow{2}{*}{$\begin{array}{l}\text { Elongation } \\
\text { at break (\%) }\end{array}$} & \multirow{2}{*}{$\begin{array}{c}\text { Young's } \\
\text { modulus (MPa) }\end{array}$} \\
\hline & $\begin{array}{c}1^{\text {st }} \\
\text { stage }\end{array}$ & $\begin{array}{c}2^{\text {nd }} \\
\text { stage }\end{array}$ & $\begin{array}{c}3^{\text {rd }} \\
\text { stage }\end{array}$ & & & & & \\
\hline COPU & 333.3 & 392.0 & 421.4 & -0.84 & 0.376 & $0.92 \pm 0.02$ & $101.0 \pm 4.3$ & $0.92 \pm 0.05$ \\
\hline COPU/MCC & 339.1 & 398.8 & 420.8 & -2.57 & 0.372 & $1.09 \pm 0.06$ & $95.2 \pm 2.2$ & $1.15 \pm 0.05$ \\
\hline $\mathrm{COPU} / \mathrm{CNC}$ & 337.7 & 400.8 & 421.1 & 1.01 & 0.404 & $1.00 \pm 0.10$ & $126.6 \pm 5.1$ & $0.79 \pm 0.04$ \\
\hline COPU/Cat. & 333.7 & 381.7 & 423.1 & -2.73 & 0.423 & $1.00 \pm 0.04$ & $82.1 \pm 9.7$ & $1.23 \pm 0.10$ \\
\hline COPU/MCC/Cat. & 336.1 & 388.9 & 421.6 & -2.88 & 0.366 & $1.85 \pm 0.09$ & $126.0 \pm 7.5$ & $1.47 \pm 0.05$ \\
\hline COPU/CNC/Cat. & 338.2 & 393.5 & 424.4 & -2.68 & 0.439 & $2.12 \pm 0.23$ & $147.4 \pm 7.4$ & $1.44 \pm 0.18$ \\
\hline
\end{tabular}


Although the reinforcement effect on the tensile strength was remarkable relative to the filler content, this mechanical property is expected to be at its highest when the filler content is above the percolation volume ${ }^{13}$. The critical volume fraction of filler necessary to reach the percolation threshold can be calculated by $\mathrm{V}_{\mathrm{TH}}=0.7(\mathrm{~L} / \mathrm{D}) \cdot{ }^{39}$ Herein, with an aspect ratio of 10 (calculated in section A from SEM images analysis) for this system the result is $\mathrm{V}_{\mathrm{TH}}=7 \%$ vol. or $10.98 \%$ wt., given the density of crystalline cellulose and the PU matrix mentioned in section B. As reported by Marcovich et al. ${ }^{40}$ simple models are available to correlate the mechanical properties of the composite with the concentration of filler, but this models are not accurate when applied in systems where the filler can react with the polyurethane matrix, as the case of the system proposed in this work. ${ }^{41}$ Without available accurate models to predict the properties of the system and taking into account that the content of filler was well below the necessary to reach the percolation volume, the effect of the addition of NCC in higher concentrations over the mechanical properties of the composite is being studied and will be the subject of future publications.

\section{E. Composite stabilities and calcifications}

TABLE III. Enzymatic degradations of PCL and the synthesized materials and degradation of the negative controls.

\begin{tabular}{ccc} 
Material & $\begin{array}{c}\text { Degradation in } \\
\text { Enzymatic medium (\%) }\end{array}$ & $\begin{array}{c}\text { Degradation of } \\
\text { Negative Controls (\%) }\end{array}$ \\
\hline \hline COPU & $0.09 \pm 0.06$ & $0.06 \pm 0.06$ \\
COPU/MCC & $0.22 \pm 0.01$ & $0.21 \pm 0.02$ \\
COPU/CNC & $0.37 \pm 0.12$ & $0.23 \pm 0.04$ \\
COPU/Cat. & $0.12 \pm 0.06$ & $0.16 \pm 0.08$ \\
COPU/MCC/Cat. & $0.38 \pm 0.03$ & $0.35 \pm 0.06$ \\
COPU/CNC/Cat. & $0.17 \pm 0.03$ & $0.14 \pm 0.07$ \\
PCL & $9.24 \pm 0.40$ & $4.20 \pm 0.16$
\end{tabular}

TABLE III shows the mass loss of the materials after 3 weeks of enzymatic degradation, and the mass loss of the control samples that were immersed in PBS without the enzyme. In contrast to the PCL, which was expected to follow a more substantial degradation in an enzymatic medium, ${ }^{42}$ the PUs and their composites showed no statistically significant difference $(\alpha>0.05)$ in terms of mass loss compared with the negative control samples during the enzymatic degradation assay. The highly 
Journal of Materials Research, 2018, 33 (17): 2598-2611. DOI: 10.1557/imr.2018.286

hydrophobic surfaces and highly packed structures of the synthesized materials may have caused the poor degradative effects of the enzyme, because these qualities hinder a proper enzymepolymer interface, which was necessary for the enzymatically-catalyzed degradation to occur. ${ }^{43}$ The $^{2}$ mass loss of the samples is thus attributed to hydrolytic non-enzymatic degradation of ester and urethane bonds in the polymers. After the 3rd week, the mass loss was very low (0.06-0.35\%), and the hydrolytic degradation assay was therefore extended to twelve weeks; the mass loss results are shown in FIG. 5 . The materials were highly resistant to hydrolytic degradation, $1.39 \pm 0.15 \%$ being the highest mass loss value, for COPU/MCC/Cat. The presence of a catalyst, cellulose, and filler particle size, all affected the material stability. Samples tended to degrade faster when cellulose was added, and more specifically mass loss was higher for the CNC than the MCC composites. This result can be attributed to the enhanced hydrophilicity of the former, as the WCA results showed. Additionally, materials in which the catalyst was added tended to show higher weight losses. This result also indicates that the catalyzed materials have a less organized structure due to the accelerated polymerization reaction, which facilitates the release of chains that break from the polymer network and their diffusion towards the medium. Other studies have correlated greater amorphous morphologies and higher free volumes to higher degradation rates. ${ }^{44}$ Therefore, COPU/CNC/Cat. was expected to be the sample most susceptible to hydrolytic degradation; nevertheless, it showed the lowest degradation value. This result could be attributed to the enhanced physical cross-linking between the cellulose nanocrystals and the HS phase of the PU matrix. A similar behavior was reported for graphene oxide/polyamide-11 composites, where changes in the hydrogen bonding behavior of a well-dispersed composite inhibited the hydrolysis process. $^{45}$ 


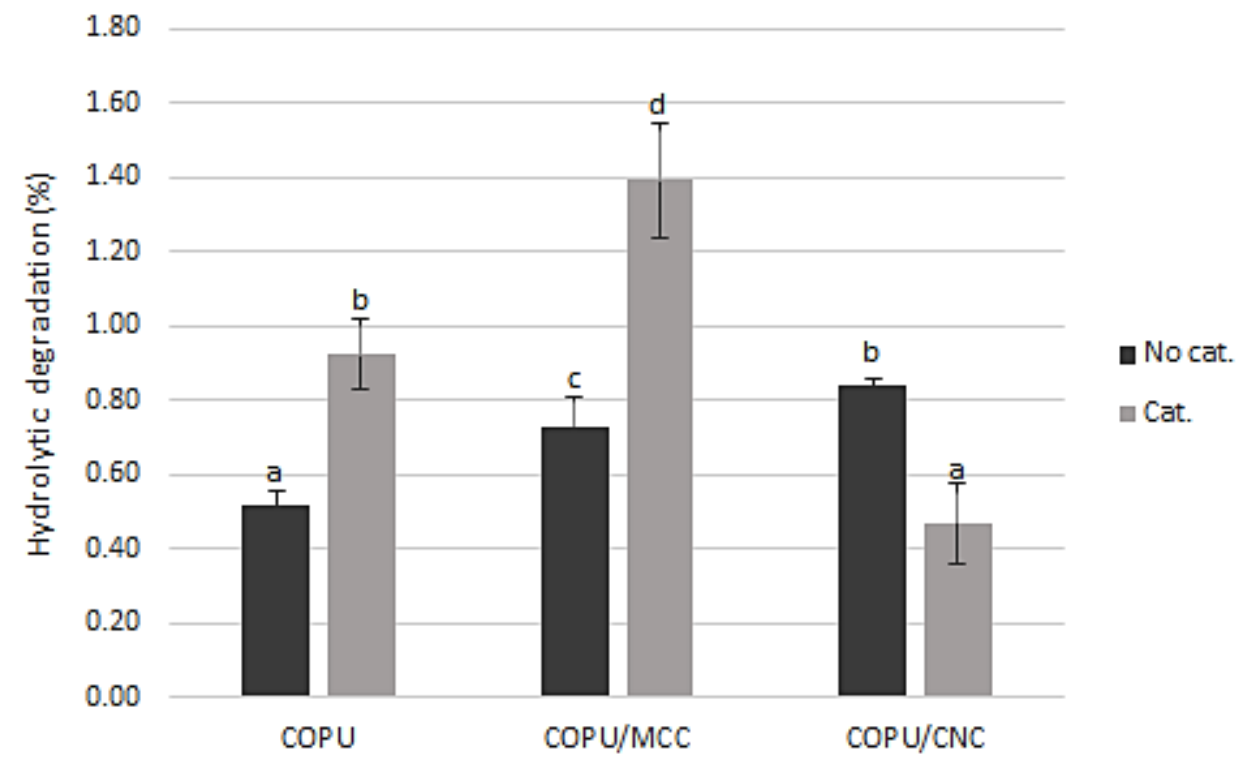

FIG. 5. Hydrolytic degradations of neat PUs and their composites after 12 weeks. Note: data are presented as the means, and error bars represent two SD $(n=4)$. Bars with different letters indicate statistically significant differences $(\alpha<0.05)$ according to the ANOVA and LSD post hoc test.

PUs can support calcium phosphate crystal formation. The polyurethane chemical composition, the hydrophilicity of the material and the presence of an ether oxygen have important effects on calcification deposition, yet the exact mechanism remains unknown. ${ }^{46}$ FIG. 6 (a) shows the SEM image of the COPU surface after immersion for 4 weeks in Golomb and Wagner's calcification medium and EDS spectra 1 and 2, which confirm the calcium phosphate nature of the observed deposits. All the samples show calcified formations on their surfaces. An average $\mathrm{Ca} / \mathrm{P}$ ratio of $1.70 \pm 0.11$ was obtained from the EDS spectra (four EDS spectra were taken per sample), which was slightly higher than the biological hydroxyapatite $\mathrm{Ca} / \mathrm{P}$ molar ratio (1.67), and in the range of 1.52 to 2.0 that has been reported in similar studies. ${ }^{47}$ FIG. 6 (b) shows the needle-like shape of the calcifications; this morphology has been reported in previous polyurethane calcification works, along with spherical, rosette-like and plaque-like deposits. ${ }^{48-50}$ Deposit morphology is believed to be affected by the physicochemical properties of the material, rather than the calcification medium used. ${ }^{48}$ 


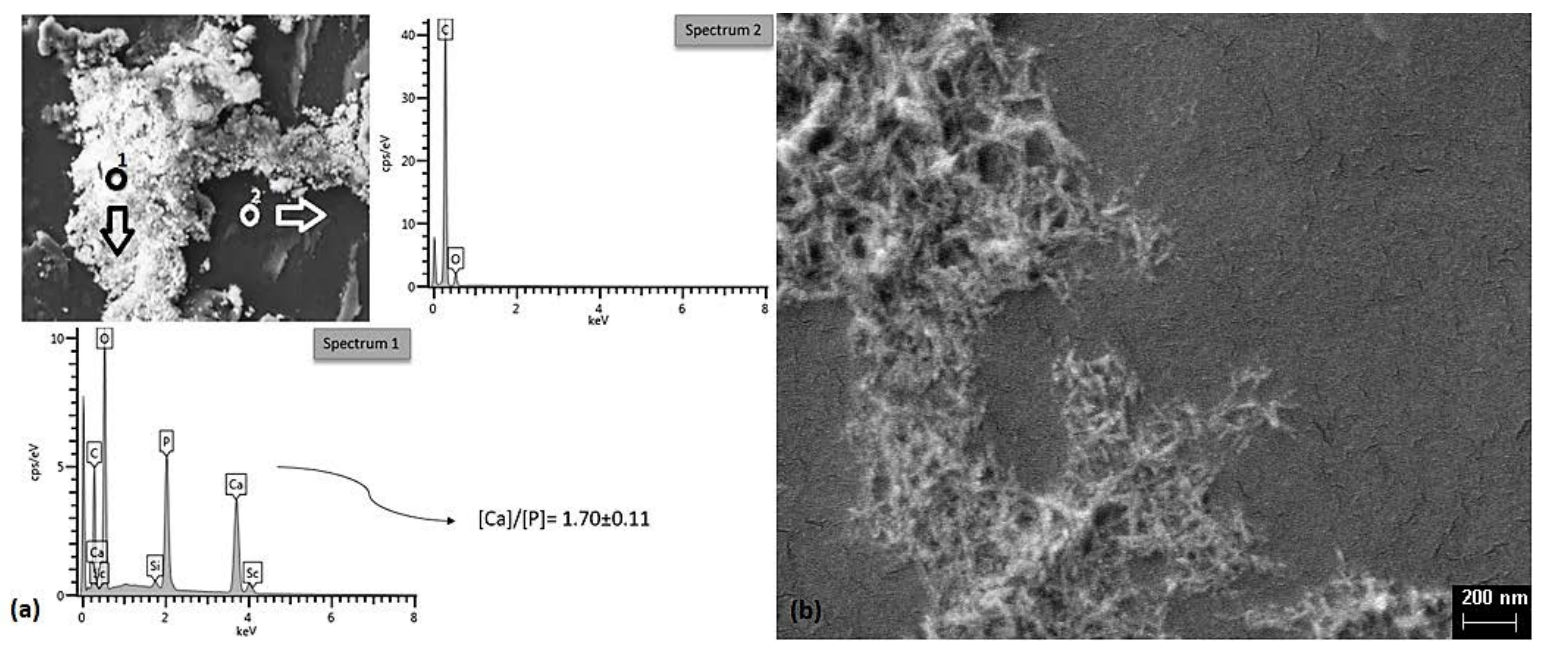

FIG. 6. (a) SEM image of the PU sample surface after immersion in the calcification medium for 4 weeks and EDS spectra confirming the calcium phosphate deposits and (b) SEM image of the needlelike calcium deposits on the PU sample surface after immersion in the calcification medium for 4 weeks.

\section{F. Fibroblast proliferation}

FIG. 7 shows the effects of the addition of cellulose, the catalyst and the rinsing protocol on L929 fibroblast viability. The statistical analysis shows significant differences $(\alpha<0.05)$ between the noncatalyzed and catalyzed samples, those with the catalyst showing a lower cell viability. The cytotoxicity of DBTDL has been reported previously, ${ }^{16}$ but it is though widely used in polyurethane synthesis pursuing biomedical applications. ${ }^{46}$ Some studies have found that DBTDL has a large effect on cell viability, leading to poor biocompatibility; ${ }^{17,51}$ however, most of them do not state any negative effect. In view of these results, the samples synthesized in this work were non-cytotoxic according to the ISO 10993-5:2009 norm "Biological evaluation of medical devices-Part 5: Tests for in vitro cytotoxicity", 52 as all materials maintained cell viability above $70 \%$ with respect to the positive control. These results suggest that these neat PUs and their composites could be suitable for applications in the biomedical field, where their ability to nucleate hydroxyapatite is mandatory and interesting. 


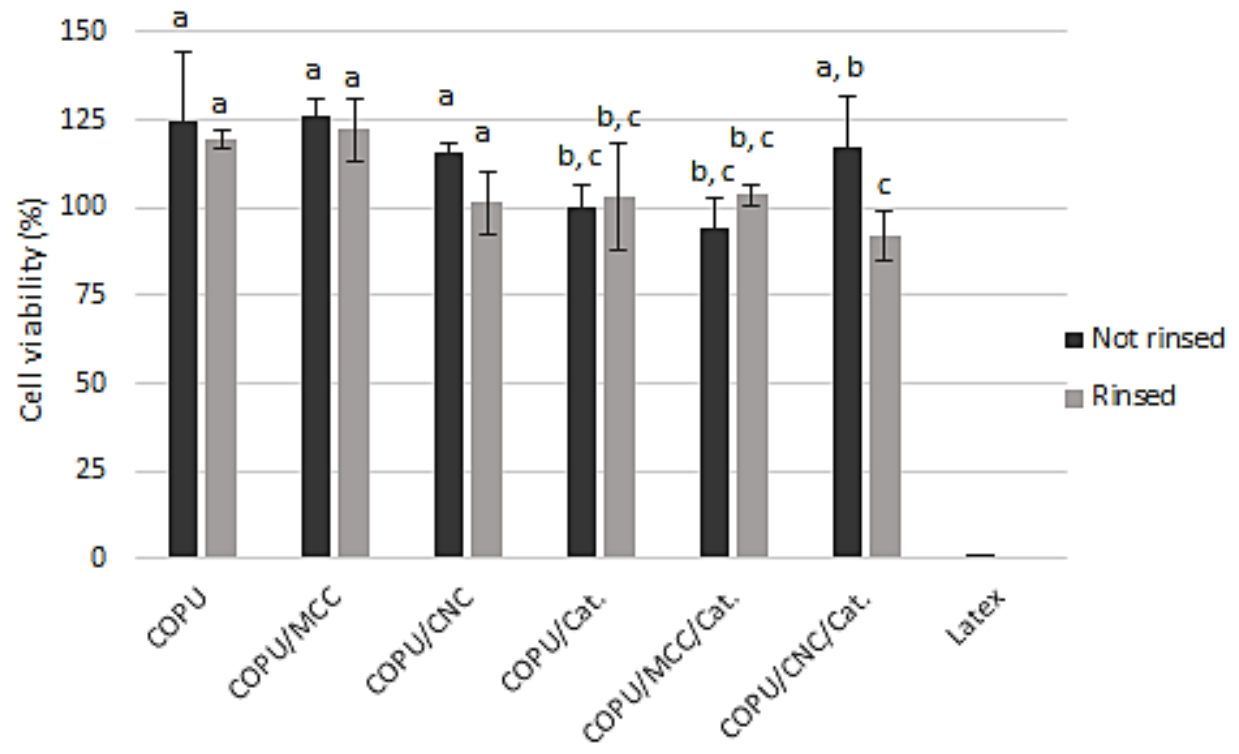

FIG. 7. Viability of L929 cells after $24 \mathrm{~h}$ of cultivation in contact with previously rinsed and not rinsed materials. Note: data are presented as the means, and error bars represent two SD ( $n=4)$. Bars with different letters indicate statistically significant differences $(\alpha<0.05)$ according to the ANOVA and LSD post hoc test.

A rinsing protocol was proposed and enacted to investigate its efficacy in avoiding cytotoxicity or reaching a higher cell viability. However, the rinsed samples did not show a better biological development and in particular the COPU/CNC/Cat. rinsed samples yielded a lower cell viability, which was attributed to the leaching of solvent trapped in the matrix after rinsing. The significant swelling of samples in contact with THF and abrupt shrinking when immersed in ethanol may have caused traces of the solvents to be trapped in the PU matrix and then its latter release when the materials where in contact with the culture medium. Adding cellulose to the materials did not have a negative effect on cell viability, confirming that the synthesis procedure is appropriate for incorporating cellulose in the form of micro or nanocrystals.

Interestingly, some of the synthesized materials (COPU, COPU/MCC, COPU/CNC and COPU/CNC/Cat.) presented cell viabilities above $100 \%$ compared with the positive control. Further research is necessary to study the factors and mechanisms that promote cell proliferation on these samples. 
Journal of Materials Research, 2018, 33 (17): 2598-2611. DOI: 10.1557/imr.2018.286

\section{CONCLUSIONS}

Cellulose composites based on a castor oil polyurethane matrix were successfully prepared by an in situ polymerization with (COPU/Cat., COPU/MCC/Cat. and COPU/CNC/Cat.) and without (COPU, COPU/MCC and COPU/CNC) the addition of DBTDL as catalyst. For the composites, containing cellulose crystals, the use of the catalyst was essential to avoid filler agglomeration, promoting the formation of a filler network, which modified the domain configurations of the materials, improving their mechanical properties, including the tensile strength and elongation at break. The addition of cellulose also improved the thermal stability of the materials, whereas the use of the catalyst decreased their soft segment degradation temperature, which was related to a decrease in the ability of the soft segments to arrange into organized domains due the accelerated polymerization reaction. These effects were confirmed by the decrease in the glass transition of the catalyzed materials. The materials were highly stable against enzymatic and hydrolytic degradation. However, the addition of cellulose and the use of the catalyst increased the hydrophilicity of the materials, increasing the susceptibility of the composites to hydrophilic degradation of ester and urethane bonds. For the COPU/CNC/Cat. combination, this particular effect differed, because these samples experienced the lowest hydrolytic degradation, despite being the most hydrophilic ones. Besides this, the increase in the mechanical properties of CNC composites, elongation at break for COPU/CNC and both elongation at break and tensile strength for COPU/CNC/Cat., suggest that a filler small particle size increase the hydrogen bond interactions between cellulose surface and PU matrix, enhancing the microphase separation and segments stability. Materials were non-cytotoxic, and a thorough rinsing after synthesis appeared not to be necessary to reach cell viabilities above $70 \%$ compared with the positive control. All samples were susceptible to calcification in simulated physiological conditions, showing needle-like depositions with a $\mathrm{Ca} / \mathrm{P}$ ratio near the physiological hydroxyapatite ratio. The mechanical, physicochemical and biological properties of the castor oil polyurethane-cellulose composites synthesized in this work with DBTDL as a catalyst suggest that this system may be an interesting candidate in the development of bioactive biomaterials.

\section{ACKNOWLEDGMENTS}

Universidad de La Sabana supported this work under grant number ING-176-2016. S.V.V. acknowledges the Universidad de La Sabana for the Teaching Assistant Scholarship for his master's studies. J.A.G.T. and A.V.L. acknowledge the support of the Spanish Ministry of Economy and Competitiveness (MINECO) through project DPI2015-65401-C3-2-R (including FEDER financial 
Journal of Materials Research, 2018, 33 (17): 2598-2611. DOI: 10.1557/imr.2018.286

support). The authors acknowledge the assistance and advice of the Electron Microscopy Service of the UPV. CIBER-BBN is an initiative funded by the VI National R\&D\&I Plan 2008-2011, Iniciativa Ingenio 2010, Consolider Program. CIBER Actions are financed by the Instituto de Salud Carlos III with assistance from the European Regional Development Fund. 


\section{SUPPLEMENTARY MATERIAL}

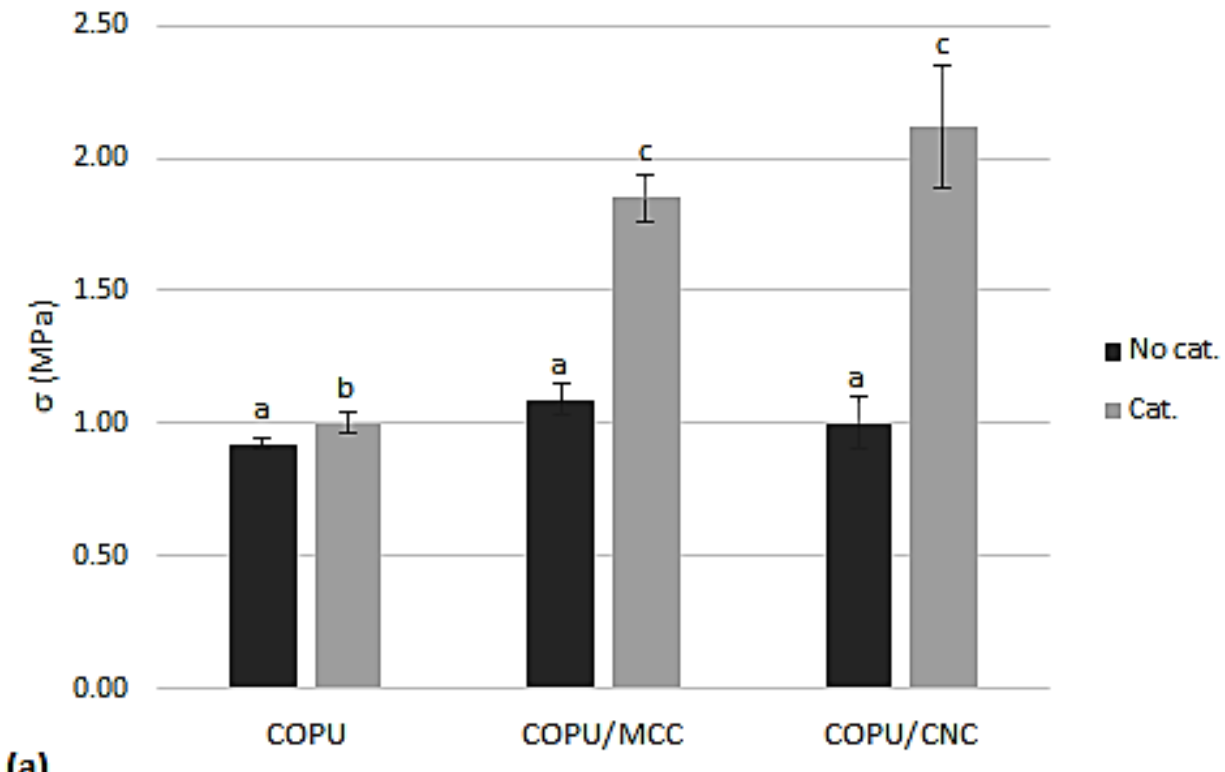

(a)

200.00

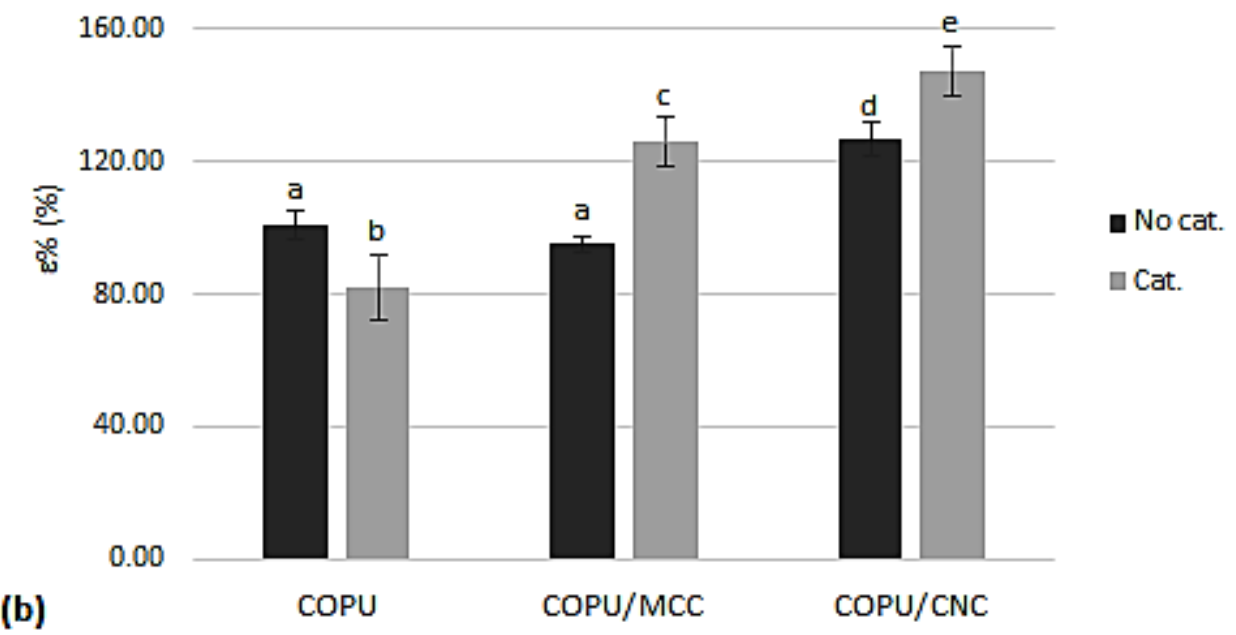

FIG. S1. (a) Tensile strengths and (b) elongations at break of PUs and their composites. Note: data are presented as the means, and error bars represent two SD $(n=4)$. Bars with different letters indicate statistically significant differences $(\alpha<0.05)$ according to the ANOVA and LSD post hoc test. 
Journal of Materials Research, 2018, 33 (17): 2598-2611. DOI: 10.1557/imr.2018.286

\section{REFERENCES}

1. M. R. Nabid and I. Omrani: Facile preparation of pH-responsive polyurethane nanocarrier for oral delivery. Mater. Sci. Eng., C 69, 532 (2016).

2. K. A. Rocco, M. W. Maxfield, C. A. Best, E. W. Dean, and C. K. Breuer: In Vivo Applications of Electrospun Tissue-Engineered Vascular Grafts: A Review. Tissue Eng., Part B 20, 628 (2014).

3. J. L. Gómez: Polymer-Ceramic Hybrid Material (Spain, 2013), WO2013178852 A1.

4. D. K. Patel, A. Biswas, and P. Maiti: Nanoparticle-induced phenomena in polyurethanes, in Adv. Polyurethane Biomater. (Elsevier Science and Technology, Cambridge, England, 2016), pp. 171.

5. B. M. Cherian, A. L. Leão, S. F. De Souza, L. M. M. Costa, G. M. De Olyveira, M. Kottaisamy, E. R. Nagarajan, and S. Thomas: Cellulose nanocomposites with nanofibres isolated from pineapple leaf fibers for medical applications. Carbohydr. Polym. 86, 1790 (2011).

6. W. Fang, S. Arola, J. M. Malho, E. Kontturi, M. B. Linder, and P. Laaksonen: Noncovalent Dispersion and Functionalization of Cellulose Nanocrystals with Proteins and Polysaccharides. Biomacromolecules 17, 1458 (2016).

7. P. Alagi, Y. J. Choi, J. Seog, and S. C. Hong: Efficient and quantitative chemical transformation of vegetable oils to polyols through a thiol-ene reaction for thermoplastic polyurethanes. Ind. Crops Prod. 87, 78 (2016).

8. T. Gurunathan, S. Mohanty, and S. K. Nayak: Isocyanate terminated castor oil-based polyurethane prepolymer : Synthesis and characterization. Prog. Org. Coat. 80, 39 (2015).

9. T. S. Omonov, E. Kharraz, and J. M. Curtis: Camelina (Camelina Sativa) oil polyols as an alternative to Castor oil. Ind. Crops Prod. 107, 378 (2017).

10. S. Oprea, V. O. Potolinca, P. Gradinariu, A. Joga, and V. Oprea: Synthesis, properties, and fungal degradation of castor-oil-based polyurethane composites with different cellulose contents. Cellulose 23, 2515 (2016).

11. A. Saralegi, M. L. Gonzalez, A. Valea, A. Eceiza, and M. A. Corcuera: The role of cellulose nanocrystals in the improvement of the shape-memory properties of castor oil-based segmented thermoplastic polyurethanes. Compos. Sci. Technol. 92, 27 (2014). 
Journal of Materials Research, 2018, 33 (17): 2598-2611. DOI: 10.1557/imr.2018.286

12. A. Kaushik and A. Garg: Castor Oil Based Polyurethane Nanocomposites with Cellulose Nanocrystallites Fillers. Adv. Mater. Res. (Durnten-Zurich, Switz.) 856, 309 (2013).

13. Z. Gao, J. Peng, T. Zhong, J. Sun, X. Wang, and C. Yue: Biocompatible elastomer of waterborne polyurethane based on castor oil and polyethylene glycol with cellulose nanocrystals. Carbohydr. Polym. 87, 2068 (2012).

14. V. Wik, M. Aranguren, and M. Mosiewicki: Castor Oil-based Polyurethanes Containing Cellulose Nanocrystals. Polym. Eng. Sci. 51, 1389 (2010).

15. J. Ryszkowska, M. Bil, P. Woźniak, M. Lewandowska-Szumieł, and K. Kurzydłowski: Influence of Catalyst Type on Biocompatibility of Polyurethanes. Mater. Sci. Forum 514-516, 887 (2006).

16. M. C. Tanzi, P. Verderio, M. G. Lampugnani, M. Resnati, E. Dejana, and E. Sturani: Cytotoxicity of some catalysts commonly used in the synthesis of copolymers for biomedical use. J. Mater. Sci.: Mater. Med. 5, 393 (1994).

17. J. G. Lundin, G. C. Daniels, C. L. Mcgann, J. Stanbro, C. Watters, M. Stockelman, and J. H. Wynne: Multi-Functional Polyurethane Hydrogel Foams with Tunable Mechanical Properties for Wound Dressing Applications. Macromol. Mater. Eng. 302, 1 (2017).

18. Á. Conejero-García, H. R. Gimeno, Y. M. Sáez, G. Vilariño-Feltrer, I. Ortuño-Lizarán, and A. Vallés-Lluch: Correlating synthesis parameters with physicochemical properties of poly(glycerol sebacate). Eur. Polym. J. 87, 406 (2017).

19. E. Rudnik, I. Resiak, and C. Wojciechowski: Thermoanalytical investigations of polyurethanes for medical purposes. Thermochim. Acta 320, 285 (1998).

20. N. D. Luong, L. H. Sinh, M. Minna, W. Jürgen, W. Torsten, S. Matthias, and S. Jukka: Synthesis and characterization of castor oil-segmented thermoplastic polyurethane with controlled mechanical properties. Eur. Polym. J. 81, 129 (2016).

21. D. Bondeson, A. Mathew, and K. Oksman: Optimization of the isolation of nanocrystals from microcrystalline cellulose by acid hydrolysis. Cellulose 13, 171 (2006).

22. J. R. Capadona, O. Van Den Berg, L. a Capadona, M. Schroeter, S. J. Rowan, D. J. Tyler, C. Weder, and O. V. a N. D. E. N. Berg: A versatile approach for the processing of polymer 
Journal of Materials Research, 2018, 33 (17): 2598-2611. DOI: 10.1557/imr.2018.286

nanocomposites with self-assembled nanofibre templates. Nat. Nanotechnol. 2, 765 (2007).

23. P. Boloori Zadeh, S. C. Corbett, and H. Nayeb-Hashemi: In-vitro calcification study of polyurethane heart valves. Mater. Sci. Eng., C 35, 335 (2014).

24. Y. Habibi and A. Dufresne: Nanocrystals from natural polysaccharides, in Handbook of Nanophysics: Nanoparticles Quantum Dots. (CRC Press, Boca Raton, Florida, USA, 2010), p. 718.

25. V. J. Dave and H. S. Patel: Synthesis and characterization of interpenetrating polymer networks from transesterified castor oil based polyurethane and polystyrene. J. Saudi Chem. Soc. 21, 18 (2017).

26. J. Datta and E. Głowińska: Effect of hydroxylated soybean oil and bio-based propanediol on the structure and thermal properties of synthesized bio-polyurethanes. Ind. Crops Prod. 61, 84 (2014).

27. C. Prisacariu: Structural studies on polyurethane elastomers, in Polyurethane Elastomers. (Springer, Vienna, Austria, 2011), pp. 23-60.

28. G. A. Senich and W. J. MacKnight: Fourier Transform Infrared Thermal Analysis of a Segmented Polyurethane. Macromolecules 13, 106 (1980).

29. C. Sun: True density of microcrystalline cellulose. J. Am. Pharm. Assoc. 94, 2132 (2005).

30. I. Yilgör, E. Yilgör, and G. L. Wilkes: Critical parameters in designing segmented polyurethanes and their effect on morphology and properties: A comprehensive review. Polymer 58, A1 (2015).

31. K. Benhamou, H. Kaddami, A. Magnin, A. Dufresne, and A. Ahmad: Bio-based polyurethane reinforced with cellulose nanofibers: A comprehensive investigation on the effect of interface. Carbohydr. Polym. 122, 202 (2015).

32. A. Santamaria-Echart, L. Ugarte, C. García-Astrain, A. Arbelaiz, M. A. Corcuera, and A. Eceiza: Cellulose nanocrystals reinforced environmentally-friendly waterborne polyurethane nanocomposites. Carbohydr. Polym. 151, 1203 (2016).

33. I. Javni, Z. S. Petrović, A. Guo, and R. Fuller: Thermal stability of polyurethanes based on vegetable oils. J. Appl. Polym. Sci. 77, 1723 (2000). 
Journal of Materials Research, 2018, 33 (17): 2598-2611. DOI: 10.1557/imr.2018.286

34. M. N. S. Kumar and Siddaramaiah: Thermogravimetric Analysis and Morphological Behavior of Castor Oil Based Polyurethane-Polyester Nonwoven Fabric Composites. J. Appl. Polym. Sci. 106, 3512 (2007).

35. S. Oprea, A. Joga, B. Zorlescu, and V. Oprea: Effect of the hard segment structure on properties of resorcinol derivatives-based polyurethane elastomers. High Perform. Polym. 26, 859 (2014).

36. S. S. Narine, X. Kong, L. Bouzidi, and P. Sporns: Physical properties of polyurethanes produced from polyols from seed oils: I. Elastomers. J. Am. Oil Chem. Soc. 84, 55 (2007).

37. S. Lin, J. Huang, P. R. Chang, S. Wei, Y. Xu, and Q. Zhang: Structure and mechanical properties of new biomass-based nanocomposite: Castor oil-based polyurethane reinforced with acetylated cellulose nanocrystal. Carbohydr. Polym. 95, 91 (2013).

38. Y. V. Yakovlev, Z. O. Gagolkina, E. V. Lobko, I. Khalakhan, and V. V. Klepko: The effect of catalyst addition on the structure, electrical and mechanical properties of the cross-linked polyurethane/carbon nanotube composites. Compos. Sci. Technol. 144, 208 (2017).

39. X. Cao, C. Xu, Y. Wang, Y. Liu, Y. Liu, and Y. Chen: New nanocomposite materials reinforced with cellulose nanocrystals in nitrile rubber. Biomacromolecules 8, 899 (2007).

40. N. E. Marcovich, M. L. Auad, N. E. Bellesi, S. R. Nutt, and M. I. Aranguren: Cellulose micro/nanocrystals reinforced polyurethane. J. Mater. Res. 21, 870 (2006).

41. N. M. Girouard, S. Xu, G. T. Schueneman, M. L. Shofner, and J. C. Meredith: Site-Selective Modification of Cellulose Nanocrystals with Isophorone Diisocyanate and Formation of Polyurethane-CNC Composites. ACS Appl. Mater. Interfaces 8, 1458 (2016).

42. J. S. Chawla and M. M. Amiji: Biodegradable poly( $\varepsilon$-caprolactone) nanoparticles for tumortargeted delivery of tamoxifen. Int. J. Pharm. (Amsterdam, Neth.) 249, 127 (2002).

43. H. Azevedo and R. Reis: Understanding the Enzymatic Degradation of Biodegradable Polymers and Strategies to Control Their Degradation Rate, in Biodegradable Systems in Tissue Engineering and Regenerative Medicine. (CRC Press, Boca Raton, Florida, USA, 2005), pp. 177-202.

44. S. Mondal and D. Martin: Hydrolytic degradation of segmented polyurethane copolymers for 
Journal of Materials Research, 2018, 33 (17): 2598-2611. DOI: 10.1557/imr.2018.286

biomedical applications. Polym. Degrad. Stab. 97, 1553 (2012).

45. S. J. A. Hocker, N. V. Hudson-Smith, P. T. Smith, C. H. Komatsu, L. R. Dickinson, H. C. Schniepp, and D. E. Kranbuehl: Graphene oxide reduces the hydrolytic degradation in polyamide- 11 . Polymer 126, 248 (2017).

46. M. Marzec, J. Kucińska-Lipka, I. Kalaszczyńska, and H. Janik: Development of polyurethanes for bone repair. Mater. Sci. Eng., C 80, 736 (2017).

47. K. Gorna and S. Gogolewski: Preparation, degradation, and calcification of biodegradable polyurethane foams for bone graft substitutes. J. Biomed. Mater. Res. 67A, 813 (2003).

48. G. Golomb and D. Wagner: Development of a new in vitro model for studying implantable polyurethane calcification. Biomaterials 12, 397 (1991).

49. Z. G. Tang, S. H. Teoh, W. McFarlane, L. A. Poole-warren, and M. Umezu: In vitro calcification of UHMWPE / PU composite membrane. Mater. Sci. Eng., C 20, 149 (2002).

50. M. Meskinfam, S. Bertoldi, N. Albanese, A. Cerri, M. C. Tanzi, R. Imani, N. Baheiraei, M. Farokhi, and S. Farè: Polyurethane foam/nano hydroxyapatite composite as a suitable scaffold for bone tissue regeneration. Mater. Sci. Eng., C 82, 130 (2018).

51. J. G. Lundin, C. L. Mcgann, G. C. Daniels, B. C. Streifel, and J. H. Wynne: Hemostatic kaolinpolyurethane foam composites for multifunctional wound dressing applications. Mater. Sci. Eng., C 79, 702 (2017).

52. International Organization for Standardization: Tests for in vitro cytotoxicity, in ISO 10993-5: Biological evaluation of medical devices. (International Organization for Standardization, Geneva, Switzerland, 2009), pp. 1-34. 